\title{
LETRAMENTO EM SAÚDE, CONHECIMENTO DA DOENÇA E RISCO PARA PÉ DIABÉTICO EM ADULTOS: ESTUDO TRANSVERSAL
}

\author{
HEALTH LITERACY, KNOWLEDGE OF DISEASE \\ AND RISK FOR DIABETIC FOOT IN ADULTS: \\ CROSS-SECTIONAL STUDY
}

\section{ALFABETIZACIÓN EN SALUD, CONOCIMIENTO DE LA ENFERMEDAD Y RIESGO DE PIE DIABÉTICO EN ADULTOS: ESTUDIO TRANSVERSAL}

\author{
Robson Giovani Paes ${ }^{1}$ \\ Maria de Fátima Mantovani ${ }^{2}$ \\ Ângela Taís Mattei da Silva ${ }^{3}$ \\ Christian Boller ${ }^{4}$ \\ Saimon da Silva Nazário 5 \\ Elaine Drehmer de Almeida Cruz ${ }^{6}$
}

Como citar este artigo: Paes RG, Mantovani MF, Silva ATM, Boller C, Nazário SS, Cruz EDA. Letramento em saúde, conhecimento da doença e risco para pé diabético em adultos: estudo transversal. Rev baiana enferm. 2022;36:e45868.

Objetivo: analisar a relação do letramento em saúde, conhecimento da doença e risco para o desenvolvimento do pé diabético em adultos com Diabetes Mellitus, de acordo com as características sociodemográficas e clínicas. Método: estudo transversal, descritivo e analítico, realizado de janeiro a março de 2020. Participaram 33 adultos com alterações glicêmicas cadastrados em unidade de saúde da Região Metropolitana de Curitiba, Paraná, Brasil. Aplicou-se questionário sociodemográfico e clínico e os instrumentos Eight-Item Health Literacy Assessment Tool e Spoken Knowledge in Low Literacy Patients with Diabetes. Resultados: houve predominância de letramento satisfatório e conhecimento adequado, associados à idade ( $\leq 59$ anos), tempo de escolaridade ( $>4$ anos) e diagnóstico ( $>5$ anos). Ao cruzar as variáveis de letramento em saúde e conhecimento da doença com o risco para pé diabético não houve significância estatística. Conclusão: níveis satisfatórios de letramento foram associados ao conhecimento adequado, sem relação com o risco para pé diabético.

Descritores: Diabetes Mellitus Tipo 2. Letramento em Saúde. Adulto. Conhecimento. Pé Diabético.

Objective: to analyze the relationship of health literacy, knowledge of the disease and risk for the development of diabetic foot in adults with Diabetes Mellitus, according to sociodemographic and clinical characteristics. Method:

\footnotetext{
Enfermeiro. Mestre em Enfermagem. Universidade Federal do Paraná. Curitiba, Paraná, Brasil. robson.paes@ufpr.br. https://orcid.org/0000-000 I-6899-4054. Enfermeira. Doutora em Enfermagem. Docente do Programa de Pós-Graduação em Enfermagem da Universidade Federal do Paraná. Curitiba, Paraná, Brasil. https://orcid.org/0000-000 I-796 I-8273.

Enfermeira. Doutora em Enfermagem. Fiscal do Conselho Regional de Enfermagem do Paraná. Londrina, Paraná, Brasil. https://orcid.org/0000-0002-4927-3806.

4 Farmacêutico. Doutor em Biotecnologia aplicada à Saúde da Criança e do Adolescente. Coordenador do curso de Graduação em Farmácia da Faculdades Pequeno Príncipe. Curitiba, Paraná, Brasil. https://orcid.org/0000-0002-1777-6695.

5 Enfermeiro. Mestre em Enfermagem. Universidade Federal do Paraná. Curitiba, Paraná, Brasil. https://orcid.org/0000-000 I-797|-7765.

Enfermeira. Doutora em Enfermagem. Docente do Programa de Pós-Graduação em Enfermagem da Universidade Federal do Paraná. Curitiba, Paraná, Brasil. https://orcid.org/0000-0002-7686-6340.
} 
cross-sectional, descriptive and analytical study, conducted from January to March 2020. The participants were 33 adults with glycemic alterations registered in a bealth unit in the Metropolitan Region of Curitiba, Paraná, Brazil. A sociodemographic and clinical questionnaire was applied and the Eight-Item Health Literacy Assessment Tool and Spoken Knowledge in Low Literacy Patients with Diabetes were applied. Results: there was a predominance of satisfactory literacy and adequate knowledge, associated with age ( $\leq 59$ years), time of schooling ( $>4$ years) and diagnosis (>5 years). When crossing the variables of health literacy and knowledge of the disease with the risk for diabetic foot there was no statistical significance. Conclusion: satisfactory levels of literacy were associated with adequate knowledge, unrelated to the risk for diabetic foot.

\section{Descriptors: Diabetes Mellitus Type 2. Literacy in Health. Adult. Knowledge. Diabetic Foot.}

Objetivo: analizar la relación de alfabetización en salud, conocimiento de la enfermedad y riesgo para el desarrollo del pie diabético en adultos con Diabetes Mellitus, según características sociodemográficas y clinicas. Método: estudio transversal, descriptivo y analítico, realizado de enero a marzo de 2020. Treinta y tres adultos con alteraciones glucémicas registrados en una unidad de salud en la Región Metropolitana de Curitiba, Paraná, Brasil, participaron en el estudio. Un cuestionario sociodemográfico y clinico y los instrumentos Eight-Item Health Literacy Assessment Tool y Spoken Knowledge in Low Literacy Patients with Diabetes. Resultados: predominó la alfabetización satisfactoria y el conocimiento adecuado, asociado a la edad ( $\leq 59$ años), tiempo de escolaridad ( $>4$ años) y diagnóstico (>5 años). Al cruzar las variables de alfabetización en salud y conocimiento de la enfermedad con el riesgo de pie diabético no bubo significación estadística. Conclusión: los niveles satisfactorios de alfabetización se asociaron con un conocimiento adecuado, no relacionado con el riesgo de pie diabético.

Descriptores: Diabetes Mellitus Tipo 2. Alfabetización en Salud. Adulto. Conocimiento. Pie Diabético.

\section{Introdução}

A Diabetes Mellitus (DM) é uma doença crônica não transmissível que representa um problema de saúde pública global, em razão de elevadas taxas de morbimortalidade e consequentes alterações micro e macro vasculares que comprometem diversos órgãos e sistemas, acarretando complicações, com destaque para o pé diabético ${ }^{(1)}$.

Esta é uma condição definida como ulceração, infecção ou destruição de partes moles, associada à doença arterial periférica e alterações neurológicas ${ }^{(2)}$. Seu impacto é significativo na qualidade de vida, mobilidade e funcionalidade física, principalmente quando não há cicatrização, culminando em indicação de amputação de segmento corporal ${ }^{(3)}$.

Dentre os fatores de risco para o desenvolvimento do pé diabético ressalta-se a Diabetes Mellitus tipo 2 (DM2), hipertensão arterial sistêmica (HAS), obesidade, consumo de tabaco, níveis aumentados de colesterol, triglicerídeos e glicose no sangue, os quais contribuem para a formação de placas de ateroma, resultando na diminuição do fluxo sanguíneo para os membros inferiores ${ }^{(4-5)}$.
Estima-se que, mundialmente, a incidência de pé diabético varia entre 5\% e 6,3\%, com prevalência de $4 \%$ a $10 \%$, em maior escala nos países em desenvolvimento ${ }^{(1)}$. No Brasil, uma análise hipotética para 7,12 milhões de pessoas com DM2 estimou que mais de 480 mil desenvolveriam úlceras, 169 mil receberiam internamento hospitalar, cerca de 81 mil necessitariam de amputação, da qual acima de 21 mil resultaria em morte ${ }^{(1)}$.

O pé diabético precede cerca de $85 \%$ das amputações não traumáticas, todavia pode ser prevenido ${ }^{(1)}$. Para tanto, a pessoa com DM deve obter o adequado conhecimento acerca da doença e níveis satisfatórios de letramento em saúde (LS), para a participação ativa no tratamento com adesão medicamentosa e mudanças do estilo de vida. Ressalta-se que o conhecimento e o LS são as bases para o desenvolvimento de aptidões para o autocuidado ${ }^{(6)}$.

O LS ou alfabetização em saúde refere-se às habilidades da pessoa e/ou da coletividade na busca, interpretação e tomada de decisão, mediante o acesso às informações e aos serviços disponíveis. Baixos níveis de LS podem 
influenciar no conhecimento da doença, na adesão ao tratamento e, consequentemente, no descontrole glicêmico, aumento dos fatores de risco e complicações ${ }^{(7-8)}$.

A mensuração de LS e do conhecimento da doença fornece subsídios para o direcionamento de ações de educação em saúde, otimizando o acesso às informações, ao desenvolvimento de habilidades e à autonomia da pessoa para o cuidado de si, com vistas a minimizar ou postergar complicações associadas a DM. Diante do exposto, o objetivo deste estudo foi analisar a relação do letramento em saúde, conhecimento da doença e risco para o desenvolvimento do pé diabético em adultos com DM, de acordo com as características sociodemográficas e clínicas.

\section{Método}

Trata-se de estudo transversal, descritivo e analítico, que seguiu as diretrizes do Strengthening the Reporting of Observational Studies in Epidemiology (STROBE) ${ }^{(9)}$. A coleta de dados ocorreu de janeiro a março de 2020 em uma unidade da Estratégia Saúde da Família (ESF), situada na Região Metropolitana de Curitiba, Paraná, Brasil, a qual foi indicada pela Coordenação de Saúde do Município por possuir o maior número de usuários cadastrados ( $n=10.141)$ e com diagnóstico de DM $(n=516)$.

A amostra foi composta por 33 adultos com DM que faziam parte da área de abrangência e estavam cadastrados na ESF onde foi realizada a coleta dos dados. Os critérios de inclusão foram: possuir idade entre 18 e 65 anos; apresentar registro em prontuário da glicemia em jejum $\geq 126 \mathrm{mg} / \mathrm{dL}$ e/ou hemoglobina glicada (HbA1c) >7\% nos anos de 2019 e 2020. Os critérios de exclusão foram: apresentar comorbidades e/ou complicações que impossibilitassem a comunicação.

A seleção dos participantes foi realizada por meio de listagem com os nomes e números cadastrais de usuários integrantes no Programa de HAS e DM (Hiperdia) da ESF, sendo realizada busca ativa em prontuário e verificado se atendiam aos critérios de elegibilidade. Dos 516 usuários com DM, foram selecionados
78, aos quais foi realizado contato telefônico para convite de participação no estudo. Não foi possível contactar 27 usuários, em razão da inexistência do número do telefone ou engano, 6 não aceitaram participar e 5 não atenderam a nenhum dos 3 telefonemas realizados em dias e horários diferentes.

Aos 40 voluntários foi proposto um encontro no domicílio ou na ESF para esclarecimento do estudo. Nesse momento, um voluntário recusou a participação, cinco apresentaram o último resultado de exame em forma impressa com glicemia de jejum e/ou HbA1c abaixo dos critérios estabelecidos para este estudo e um apresentou dificuldade na comunicação devido ao diagnóstico de Alzheimer, resultando em amostra final de 33 usuários.

Para os que aceitaram participar do estudo foi realizada a leitura do Termo de Consentimento Livre e Esclarecido (TCLE). Após assinatura, iniciou-se a consulta de enfermagem, conduzida por um único pesquisador, que aplicou o questionário sociodemográfico e clínico e os instrumentos Eight-Item Health Literacy Assessment Tool (HLAT-8) e Spoken Knowledge in Low Literacy Patients with Diabetes (SKILLD), para avaliação do LS e do conhecimento sobre a DM. A consulta teve duração média de 90 minutos, sendo que 31 foram realizadas em domićlio e 2 em consultório da ESF, empregando-se protocolo único e equipamentos padronizados.

O questionário sociodemográfico e clínico foi previamente testado e validado pelo grupo de pesquisas em saúde do adulto e idoso, do qual os autores fazem parte, e continha questões referentes ao sexo, idade, escolaridade, renda, tempo de diagnóstico de DM, quantidade de medicações diárias, atividade física, índice de massa corporal (IMC) e as avaliações para o rastreamento do pé diabético (alterações ósseas nos pés, valores do índice tornozelo-braquial (ITB) e testes de sensibilidade plantar e vibratória).

A classificação de risco para pé diabético foi realizada com base nos achados clínicos por meio da avaliação de sensibilidade tátil mediante o uso de estesiômetro de Semmes-Weistein de $10 \mathrm{~g}$ $\left(\right.$ Sorri-Bauru $\left.^{\circledR}\right)$, sensibilidade vibratória com diapasão $128 \mathrm{~Hz}\left(\right.$ Golgran $\left.^{\circledR}\right)$ e pelo ITB. Foi interpretado 
como perda da sensibilidade protetora dos pés quando o participante não sentiu duas das três aplicações do monofilamento de $10 \mathrm{~g}$ nas superfícies das falanges distais do primeiro, terceiro e quinto dedo, e nos três pontos dos metatarsos dos respectivos dedos. A sensibilidade vibratória foi avaliada como anormal quando o participante respondeu de forma incorreta duas das três aplicações do diapasão na região maleolar medial e/ ou na falange proximal do hálux ${ }^{(10)}$.

A obtenção do ITB ocorreu pela verificação da pressão arterial sistólica (PAS) no braço e na perna, com uso de esfigmomanômetro aneroide calibrado (Premium ${ }^{\circledR}$ ) e doppler vascular portátil (DV 610B Medmega ${ }^{\circledR}$ ). O valor foi obtido pela razão entre a PAS braquial dividida pela PAS do tornozelo, classificado em normal (1,01 a 1,40mmHg), limítrofe $(0,91$ a $1,00 \mathrm{mmHg})$ e anormal $(<0,90 \mathrm{mmHg})^{(11)}$.

O risco para o desenvolvimento do pé diabético foi categorizado em 0 , quando não havia perda de sensibilidade e ITB normal; risco 1 quando havia alterações de sensibilidade com ou sem deformidades nos pés; risco 2 quando ITB $<0,90 \mathrm{mmHg}$ com ou sem perda da sensibilidade; e risco 3 quando histórico de amputação ou ulceração ${ }^{(10)}$. Para análise desses dados, os valores foram recategorizados, sendo considerado baixo/moderado risco para o desenvolvimento de pé diabético quando índices 0 e 1 e alto risco, quando 2 e 3.

A HLAT-8 foi utilizada para avaliar os níveis de LS. A versão traduzida e validada para o português é composta de 8 questões, com pontuação entre 0 e 37; quanto maior a pontuação melhor a avaliação do item correspondente. Essa escala permite a mensuração de quatro fatores estruturais: entendimento das informações de saúde (EIS: questões 1 e 2; de zero a cinco pontos); busca de informações em saúde (BIS: questões 3 e 4; de zero a quatro pontos); interação em saúde (IS: questões 5 e 6; de zero a cinco pontos) e conhecimento crítico em saúde (CCS: questões 7 e 8; com pontuação máxima de cinco e quatro pontos, respectivamente) ${ }^{(12)}$. Para este estudo considerou-se a pontuação máxima da HLAT-8 de 37 pontos, adotando-se como LS satisfatório a pontuação total $\geq 50 \%$ ( $\geq 19$ pontos); os valores menores foram considerados como LS insatisfatório.

O SKILLD foi utilizado para avaliar o conhecimento da DM; a versão traduzida e validada para o português do Brasil é composta por 10 questões com pontuação que varia de 0 a 100\%. Esse instrumento aceita respostas verbais, não sendo necessário ao participante ter habilidades de leitura. No caso da não compreensão da questão, utiliza-se o segundo enunciado com a questão reformulada ${ }^{(13)}$. Para análise dos valores obtidos no SKILLD considerou-se como conhecimento adequado a pontuação total $\geq 6$ ( $>50 \%$ ); as menores foram categorizadas como conhecimento inadequado.

Os dados foram digitados em planilhas do Microsoft Excel 365®, com dupla conferência e exportados posteriormente para o software Statistical Package for the Social Science (SPSS), versão $20.0 \AA$, por meio do qual realizou-se a análise estatística. Analisou-se os resultados da HLAT-8 de forma descritiva por medidas de tendência central - média e desvio-padrão (DP). Os erros e acertos do SKILLD foram analisados por frequência simples (n) e absoluta (\%).

Empregou-se a análise de confiabilidade pelo alfa de Cronbach para a HLAT-8 e o SKILLD, em razão destes instrumentos serem validados para o português do Brasil em populações diferentes da elencada neste estudo.

As variáveis foram categorizadas e recategorizadas por parâmetros estabelecidos pelos pesquisadores com base na literatura, sendo: idade ( $\leq 59$ e >59 anos), classificada em adultos e idosos; tempo de diagnóstico (até 5 e $>5$ anos), em razão das experiências decorrentes da doença; escolaridade (até 4 e >4anos), considerando ensino fundamental (baixa escolaridade). Para essa análise utilizou-se os dados de 32 participantes); renda $(\leq \mathrm{R} \$ 1.045,00$ e $>\mathrm{R} \$ 1.045,00)$, baseada no salário mínimo brasileiro vigente no período do estudo; sexo (masculino e feminino); nível de LS (satisfatório e insatisfatório) e conhecimento (adequado e inadequado), com base nas pontuações estabelecidas na validação dos instrumentos ${ }^{(12-13)}$; e risco para pé diabético, de acordo com a classificação de risco, conforme definição da literatura (risco 0 e 1 (baixo/moderado) e 2 e 3 (alto) $)^{(10)}$. 
Para análise da relação das variáveis aplicou-se o teste de qui-quadrado com correção de Yates, e, quando estas apresentaram distribuição em uma das células com menos de cinco participantes, utilizou-se o teste Exato de Fisher. Adotou-se como significativo um valor de $\mathrm{p} \leq 0,005$.

O estudo foi aprovado pelo Comitê de Ética em Pesquisa (CEP) da Universidade Federal do Paraná (UFPR), sob Parecer nº 3.752.041 e Certificado de Apresentação de Apreciação Ética (CAAE) 20244119.3.0000.0102. Respeitou-se todas as diretrizes éticas especificadas na Resolução $\mathrm{n}^{\mathrm{o}}$ 466, de 12 de dezembro de 2012, do Conselho Nacional de Saúde, para pesquisas envolvendo seres humanos.

\section{Resultados}

Os participantes possuíam idade mínima de 34 anos e máxima de $65(57,0 \pm 8,08)$. Quanto à idade, 84,8\% $(n=28)$ tinham idade $>59$ anos; $78,8 \% \quad(n=26)$ tinham tempo de escolaridade $>4$ anos, 69,7\% $(n=23)$ eram do sexo feminino e $69,7 \% \quad(n=23)$ possuíam renda per capita menor ou igual a um salário mínimo nacional ( R\$ 1.045,00).

Com relação às variáveis clínicas, 91,0\% $(n=30)$ não praticavam atividade física regularmente, 75,7\% $(n=25)$ possuíam DM há >5 anos, $57,6 \%(n=19)$ apresentavam alto risco para o desenvolvimento de pé diabético, 54,5\% (n=18) utilizavam cinco ou menos medicamentos de forma contínua e 51,5\% (n=17) apresentavam IMC $\leq 29,9 \mathrm{~kg} / \mathrm{m}^{2}$.

No que tange à mensuração do LS, a média de pontuação da HLAT-8 foi de 20,7 $\pm 5,4$, com mínima de 9 e máxima de 32, tendo $57,6 \%(n=19)$ obtido níveis satisfatórios de LS. As notas médias das questões e dos fatores estruturais da HLAT-8 estão detalhadas na Tabela 1.

Tabela 1 - Avaliação do Letramento em Saúde de adultos com Diabetes Mellitus. Região Metropolitana de Curitiba, Paraná, Brasil - 2020. (N=33)

\begin{tabular}{|c|c|c|}
\hline Variáveis & Média & Desvio-padrão \\
\hline \multicolumn{3}{|l|}{ Questões da Eight-Item Health Literacy Assessment Tool } \\
\hline $\begin{array}{l}\text { Q1 - Quanto você compreende das instruções nas bulas de } \\
\text { medicamentos? }\end{array}$ & 1,6 & 1,7 \\
\hline $\begin{array}{l}\text { Q2 - Quanto você entende sobre informações de saúde em } \\
\text { folhetos/cartilhas? }\end{array}$ & 2,2 & 1,8 \\
\hline $\begin{array}{l}\text { Q3 - Quando eu tenho dúvidas sobre doenças ou queixas, eu sei onde } \\
\text { posso encontrar estas informações. }\end{array}$ & 2,7 & 0,7 \\
\hline $\begin{array}{l}\text { Q4 - Quando eu quero fazer algo para a minha saúde sem estar doente, } \\
\text { eu sei onde posso encontrar estas informações. }\end{array}$ & 2,2 & 1,1 \\
\hline $\begin{array}{l}\text { Q5 - Com que frequência você conseguiu ajudar seus familiares ou um } \\
\text { amigo, caso eles tenham tido dúvidas sobre problemas de saúde? }\end{array}$ & 3,7 & 1,6 \\
\hline $\begin{array}{l}\text { Q6 - Quando você teve dúvidas sobre problemas e questões de saúde, } \\
\text { quantas vezes você conseguiu receber conselhos e informações de } \\
\text { outras pessoas (familiares e amigos)? }\end{array}$ & 3,3 & 1,8 \\
\hline $\begin{array}{l}\text { Q7 - Como você acredita que sabe escolher os conselhos e } \\
\text { recomendações que sejam melhores para a sua saúde? }\end{array}$ & 3,6 & 0,8 \\
\hline $\begin{array}{l}\text { Q8 - Em relação às informações sobre saúde na Internet, eu sou capaz } \\
\text { de determinar quais fontes são de alta ou de baixa qualidade. }\end{array}$ & 1,5 & 1,5 \\
\hline \multicolumn{3}{|l|}{ Fatores estruturais da Eight-Item Health Literacy Assessment Tool } \\
\hline Entendimento das informações de saúde & 3,8 & 2,9 \\
\hline Busca de informação em saúde & 4,9 & 1,2 \\
\hline Interação em saúde & 7,0 & 2,5 \\
\hline Conhecimento crítico em saúde & 5,1 & 1,8 \\
\hline
\end{tabular}

Fonte: Elaboração própria.

Em relação à aplicação do SKILLD, 54,4\% $(n=18)$ apresentaram conhecimento adequado sobre a DM, e a pontuação variou de 0 a 100\%, com média de acertos 5,2 $\pm 2,6$. A percentagem de erros e acertos do instrumento é apresentada na Tabela 2. 
Tabela 2 - Percentagem de erros e acertos dos adultos com Diabetes Mellitus no instrumento Spoken Knowledge in Low Literacy Patients with Diabetes. Região Metropolitana de Curitiba, Paraná, Brasil 2020. ( $N=33)$

\begin{tabular}{|c|c|c|c|c|}
\hline \multirow{2}{*}{$\begin{array}{l}\text { Questões do Spoken Knowledge in Low Literacy Patients with } \\
\text { Diabetes }\end{array}$} & \multicolumn{2}{|c|}{$\mathbf{n}$} & \multicolumn{2}{|c|}{$\%$} \\
\hline & Erro & Acerto & Erro & Acerto \\
\hline Q1 - Quais são os sinais e sintomas da glicemia alta? & 28 & 5 & 84,8 & 15,2 \\
\hline Q2 - Quais são os sinais e sintomas da glicemia baixa? & 22 & 11 & 66,7 & 33,3 \\
\hline Q3 - Como dever ser tratada a glicemia baixa? & 8 & 25 & 24,2 & 75,8 \\
\hline $\begin{array}{l}\text { Q4 - Com que frequência uma pessoa que tem diabetes deve } \\
\text { examinar os pés? }\end{array}$ & 21 & 12 & 63,6 & 36,4 \\
\hline $\begin{array}{l}\text { Q5 - Por que o exame dos pés é importante para uma pessoa que } \\
\text { tem diabetes? }\end{array}$ & 13 & 20 & 39,4 & 60,6 \\
\hline $\begin{array}{l}\text { Q6 - Com que frequência uma pessoa com diabetes deve consultar } \\
\text { o oculista e por que isso é importante? }\end{array}$ & 9 & 24 & 27,3 & 72,7 \\
\hline Q7 - Qual é a glicemia de jejum normal? & 8 & 25 & 24,2 & 75,8 \\
\hline Q8 - Qual é o valor normal da hemoglobina glicada? & 21 & 12 & 63,6 & 36,4 \\
\hline $\begin{array}{l}\text { Q9 - Quantas vezes por semana uma pessoa com diabetes deve } \\
\text { fazer exercício e por quanto tempo? }\end{array}$ & 15 & 18 & 45,5 & 54,5 \\
\hline $\begin{array}{l}\text { Q10 - Quais são as complicações de longo prazo do diabetes } \\
\text { descontrolado? }\end{array}$ & 17 & 16 & 51,5 & 48,5 \\
\hline
\end{tabular}

Fonte: Elaboração própria.

Ao avaliar a confiabilidade dos instrumentos HLAT-8 e SKILLD, obteve-se valor do alfa de Cronbach de 0,511 e 0,756, respectivamente. Ao correlacionar as variáveis sociodemográficas e clínicas com os níveis de conhecimento da doença, LS e risco para pé diabético, identificou-se associação da LS com o conhecimento da doença ( $\mathrm{p}=0,015)$, sendo que pessoas com nível de LS adequado também apresentaram conhecimento satisfatório $(n=14 ; 77,8 \%)$. Entretanto, não houve significância estatística entre o nível de conhecimento e LS com o risco para pé diabético (Tabela 3).

Tabela 3 - Relação entre as variáveis sociodemográficas e clínicas com o conhecimento da Diabetes Mellitus, nível de letramento em saúde e risco para o pé diabético. Região Metropolitana de Curitiba, Paraná, Brasil - 2020. (N=33)

\begin{tabular}{|c|c|c|c|c|c|c|}
\hline \multirow[t]{2}{*}{ Variáveis } & \multicolumn{3}{|c|}{$\begin{array}{c}\text { Conhecimento da Diabetes } \\
\text { Mellitus (Spoken Knowledge in } \\
\text { Low Literacy Patients with Diabetes) }\end{array}$} & \multicolumn{3}{|c|}{$\begin{array}{c}\text { Nivel de Letramento em Saúde } \\
\text { (Eight-Item Health Literacy } \\
\text { Assessment Tool) }\end{array}$} \\
\hline & $\begin{array}{c}\text { Adequado } \\
\mathrm{n}(\%)\end{array}$ & $\begin{array}{c}\text { Inadequado } \\
\mathrm{n}(\%)\end{array}$ & p-valor & $\begin{array}{c}\text { Satisfatório } \\
\mathrm{n}(\%)\end{array}$ & $\begin{array}{c}\text { Insatisfatório } \\
\mathrm{n}(\%) \\
\end{array}$ & p-valor \\
\hline Sexo & & & $1,00(1)$ & & & 0,335 (1) \\
\hline Feminino & $13(56,5)$ & $10(43,5)$ & & $15(65,2)$ & $8(34,8)$ & \\
\hline Masculino & $5(50,0)$ & $5(50,0)$ & & $4(40,0)$ & $6(60,0)$ & \\
\hline Faixa etária (anos) & & & 0,238 (1) & & & $0,421(1)$ \\
\hline$\leq 59$ & $12(66,7)$ & $6(33,3)$ & & $12(66,7)$ & $6(33,3)$ & \\
\hline$>59$ & $6(40,0)$ & $9(60,0)$ & & $7(46,7)$ & $8(53,3)$ & \\
\hline Escolaridade (anos) & & & $0,365(2)$ & & & $0,194(2)$ \\
\hline$\leq 4$ & $2(33,3)$ & $4(66,7)$ & & $2(33,3)$ & $4(66,7)$ & \\
\hline$>4$ & $16(61,5)$ & $10(38,5)$ & & $17(65,4)$ & $9(34,6)$ & \\
\hline $\begin{array}{l}\text { Renda per capita } \\
\text { (reais) (3) }\end{array}$ & & & $0,070(2)$ & & & $0,707(2)$ \\
\hline$\leq 1.045,00$ & $10(43,5)$ & $13(56,5)$ & & $14(60,9)$ & $9(39,1)$ & \\
\hline$>1.045,00$ & $8(80,0)$ & $2(20,0)$ & & $5(50,0)$ & $5(50,0)$ & \\
\hline
\end{tabular}


Tabela 3 - Relação entre as variáveis sociodemográficas e clínicas com o conhecimento da Diabetes Mellitus, nível de letramento em saúde e risco para o pé diabético. Região Metropolitana de Curitiba, Paraná, Brasil - 2020. (N=33)

\begin{tabular}{|c|c|c|c|c|c|c|}
\hline \multirow[t]{2}{*}{ Variáveis } & \multicolumn{3}{|c|}{$\begin{array}{c}\text { Conhecimento da Diabetes } \\
\text { Mellitus (Spoken Knowledge in } \\
\text { Low Literacy Patients with Diabetes) }\end{array}$} & \multicolumn{3}{|c|}{$\begin{array}{c}\text { Nivel de Letramento em Saúde } \\
\text { (Eight-Item Health Literacy } \\
\text { Assessment Tool) }\end{array}$} \\
\hline & \begin{tabular}{|c} 
Adequado \\
$\mathrm{n}(\%)$
\end{tabular} & $\begin{array}{c}\text { Inadequado } \\
\mathrm{n}(\%)\end{array}$ & p-valor & $\begin{array}{c}\text { Satisfatório } \\
\mathrm{n}(\%)\end{array}$ & $\begin{array}{c}\text { Insatisfatório } \\
\mathrm{n}(\%)\end{array}$ & p-valor \\
\hline $\begin{array}{l}\text { Tempo de diagnóstico } \\
\text { (anos) }\end{array}$ & \multicolumn{5}{|c|}{$0,101(2)$} & \multirow[t]{3}{*}{$0,238(2)$} \\
\hline$\leq 5$ & $2(25,0)$ & $6(75,0)$ & & $3(37,5)$ & $5(62,5)$ & \\
\hline$>5$ & $16(64,0)$ & $9(36,0)$ & & $16(64,0)$ & $9(36,0)$ & \\
\hline Medicações diárias & & & 0,187 (1) & & & $0,073(2)$ \\
\hline$\leq 5$ & $8(42,1)$ & $11(57,9)$ & & $8(42,1)$ & $11(57,9)$ & \\
\hline$>5$ & $10(71,4)$ & $4(28,6)$ & & $11(78,6)$ & $3(21,4)$ & \\
\hline Atividade física & & & $0,579(2)$ & & & $1,000(2)$ \\
\hline Regular & $1(33,3)$ & $2(66,7)$ & & $2(66,7)$ & $1(33,3)$ & \\
\hline $\begin{array}{l}\text { Esporádica ou não } \\
\text { praticante }\end{array}$ & $17(56,7)$ & $13(43,3)$ & & $17(56,7)$ & $13(43,3)$ & \\
\hline Índice de Massa & & & $0,589(1)$ & & & $0,616(1)$ \\
\hline \multicolumn{7}{|l|}{ Corporal $\left(\mathrm{kg} / \mathrm{m}^{2}\right)$} \\
\hline$\leq 29,9$ & $8(47,1)$ & $9(52,9)$ & & $11(64,7)$ & $6(35,3)$ & \\
\hline$\geq 30$ & $10(62,5)$ & $6(37,5)$ & & $8(50,0)$ & $8(50,0)$ & \\
\hline $\begin{array}{l}\text { Risco para o pé } \\
\text { diabético }\end{array}$ & & & $0,421(1)$ & & & $0,690(1)$ \\
\hline Baixo/ moderado & $6(42,9)$ & $8(57,1)$ & & $7(50,0)$ & $7(50,0)$ & \\
\hline Alto & $12(63,2)$ & $7(36,8)$ & & $12(63,2)$ & $7(36,8)$ & \\
\hline \multicolumn{7}{|l|}{$\begin{array}{l}\text { Saúde (Eight-Item Health } \\
\text { Literacy Assessment Tool) }\end{array}$} \\
\hline Satisfatório & $14(77,8)$ & $4(22,2)$ & & - & - & \\
\hline Insatisfatório & $5(33,3)$ & $10(66,7)$ & & - & - & \\
\hline
\end{tabular}

Fonte: Elaboração própria.

Nota: Sinal convencional utilizado:

- Dado numérico igual a zero não resultante de arredondamento.

(1) Teste de qui-quadrado com correção de Yates.

(2) Teste exato de Fisher.

(3) Salário mínimo brasileiro em janeiro e março de 2020 era de R\$1.045,00.

Ao analisar as variáveis sociodemográficas e clínicas com o risco para pé diabético, verificou-se que os participantes com alto risco, obtinham $>4$ anos de escolaridade $(n=17 ; 65,4 \%)$, não praticavam atividade física $(\mathrm{n}=17 ; 56,7 \%) \mathrm{e}$ possuíam renda per capita $\leq \mathrm{R} \$ 1.045,00 \quad(\mathrm{n}=17$; 65,4\%) (Tabela 4).

Tabela 4 - Relação entre as variáveis sociodemográficas e clínicas com o risco para o pé diabético. Região Metropolitana de Curitiba, Paraná, Brasil - 2020. (N=33)

\begin{tabular}{l|c|c|c}
\hline \multirow{2}{*}{ Variáveis } & \multicolumn{3}{|c}{ Risco para o pé diabético } \\
\cline { 2 - 4 } & $\begin{array}{c}\text { Baixo/moderado } \\
\mathrm{n}(\%)\end{array}$ & $\begin{array}{c}\text { Alto risco } \\
\mathrm{n}(\%)\end{array}$ & $\mathrm{p}$-valor \\
\hline Sexo & & & $0,455(2)$ \\
Feminino & $11(47,8)$ & $12(52,2)$ & \\
Masculino & $3(30,0)$ & $7(70,0)$
\end{tabular}


Tabela 4 - Relação entre as variáveis sociodemográficas e clínicas com o risco para o pé diabético. Região Metropolitana de Curitiba, Paraná, Brasil - 2020. (N=33)

\begin{tabular}{|c|c|c|c|}
\hline \multirow[b]{2}{*}{ Variáveis } & \multicolumn{3}{|c|}{ Risco para o pé diabético } \\
\hline & $\begin{array}{c}\text { Baixo/moderado } \\
\text { n (\%) }\end{array}$ & $\begin{array}{c}\text { Alto risco } \\
\mathrm{n}(\%)\end{array}$ & p-valor \\
\hline Faixa etária (anos) & & & $0,923(1)$ \\
\hline$\leq 59$ & $7(38,9)$ & $11(61,1)$ & \\
\hline$>59$ anos & $7(46,7)$ & $8(53,3)$ & \\
\hline Escolaridade (anos) & & & $0,194(2)$ \\
\hline$\leq 4$ & $4(66,7)$ & $2(33,3)$ & \\
\hline$>4$ & $9(34,6)$ & $17(65,4)$ & \\
\hline Renda per capita (reais)(3) & & & $0,257(2)$ \\
\hline$\leq \mathrm{R} \$ 1.045,00$ & $8(34,8)$ & $15(65,2)$ & \\
\hline$>\mathrm{R} \$ 1.045,00$ & $6(60,0)$ & $4(40,0)$ & \\
\hline Tempo de diagnóstico (anos) & & & $0,695(2)$ \\
\hline$\leq 5$ & $4(50,0)$ & $4(50,0)$ & \\
\hline$>5$ & $10(40,0)$ & $15(60,0)$ & \\
\hline Medicações diárias & & & $0,073(2)$ \\
\hline$\leq 5$ & $11(57,9)$ & $8(42,1)$ & \\
\hline$>5$ & $3(21,4)$ & $11(78,6)$ & \\
\hline Atividade física & & & $1,000(2)$ \\
\hline Regular & $1(33,3)$ & $2(66,7)$ & \\
\hline Esporádica ou não praticante & $13(43,3)$ & $17(56,7)$ & \\
\hline Índice de Massa Corporal $\left(\mathrm{kg} / \mathrm{m}^{2}\right)$ & & & $0,616(1)$ \\
\hline$\leq 29,9$ & $6(35,3)$ & $11(64,7)$ & \\
\hline$\geq 30$ & $8(50,0)$ & $8(50,0)$ & \\
\hline
\end{tabular}

Fonte: Elaboração própria.

(1) Teste de qui-quadrado com correção de Yates.

(2) Teste exato de Fisher.

(3) Salário mínimo brasileiro em janeiro e março de 2020 era de $\mathrm{R} \$ 1.045,00$.

\section{Discussão}

Neste estudo objetivou-se analisar a relação do LS, conhecimento da doença e risco para o desenvolvimento de pé diabético de acordo com as características sociodemográficas e clínicas de adultos com DM. Identificou-se que os participantes com conhecimento adequado apresentavam maior nível de LS $(p=0,015)$, e estes resultados foram predominantes nas pessoas com idade $\leq 59$ anos, tempo de escolaridade $>4$ anos e diagnóstico de DM >5 anos. Todavia, não houve relação dessas variáveis com o risco para pé diabético.

Esses achados são similares à pesquisa transversal realizada em uma clínica de DM na cidade de Khaf, Sudeste do Irã, com 404 participantes com DM2, que demonstrou relação entre LS e conhecimento da doença associado à idade e ao nível educacional, os quais correlacionavam-se positivamente ao comportamento de autocuidado com os pés ${ }^{(6)}$.

Outra pesquisa transversal, realizada no Sul da Tasmânia, aplicou, para 222 participantes com DM, o The Short Test of Functional Health Literacy in Adults (S-TOFHLA) e um questionário de conhecimento sobre a doença. Realizou também inspeção nos pés, para identificação de fatores de risco, apontando que as pessoas mais idosas possuíam alto risco para pé diabético, baixos níveis de LS e conhecimento inadequado sobre a doença, corroborando os achados desta pesquisa. Em análise univariada, o aumento da pontuação no questionário de LS estava associado a $4 \%$ de menor chance para alto risco de pé diabético (Odds Ratio 0,96, IC 95\% 0,93 a 0,99 ), porém quando cruzados com as variáveis idade, sexo, perda da sensibilidade protetora, doença arterial periférica e deformidade óssea nos pés, não houve significância estatística ${ }^{(14)}$. 
Ao analisar descritivamente o risco para pé diabético com a idade $\leq 59$ anos, observou-se que $61,1 \%(n=11)$ dos participantes nessa faixa etária tinham alto risco, mesmo com conhecimento adequado e nível de LS satisfatório. Esse fator pode estar associado ao baixo controle glicêmico, além do desconhecimento de alguns aspectos da doença, como os observados nas questões menos pontuadas do SKILLD (Tabela 2), referentes aos sinais e sintomas de hiper e hipoglicemia, valor de normalidade da Hb1Ac e a frequência do exame nos pés. Na pesquisa de validação do SKILLD para a versão em espanhol, com 111 hispano-americanos, as questões relativas aos sinais e sintomas da hiperglicemia (questão 1; 17,1\%) e valor normal da HbA1c (questão 8; 18,0\%) também obtiveram as menores pontuações ${ }^{(15)}$.

Outros fatores observados que podem influenciar no descontrole da DM, inadequado conhecimento e aumento do risco para pé diabético, foram as médias baixas de LS nos fatores estruturais EIS e BIS (Tabela 1), principalmente na questão alusiva à compreensão das informações nas bulas de medicamentos, que pode contribuir para a falha da adesão ao tratamento. Contrapondo-se a esse resultado, pesquisa de validação da HLAT-8 para a língua chinesa com 650 adolescentes apresentou a maior média na questão da compreensão das informações nas bulas $(3,83 \pm 1,04)^{(16)}$.

Neste estudo, notou-se que os participantes que utilizavam $\leq 5$ medicações diárias apresentavam menores níveis de LS e de conhecimento da doença comparados com aqueles que usavam $>5$ medicações/dia (11; 57,9\% versus 8; 42,1\% em ambas as variáveis) (Tabela 3). Cabe salientar que não foi o objetivo deste estudo avaliar a adesão medicamentosa, mas, sim, analisar se a quantidade de medicamentos/dia tinha relação com o conhecimento da doença, LS e risco para pé diabético.

A questão relativa à capacidade de determinar informações de alta e baixa qualidade na Internet teve a menor média da escala (Tabela 1). Esse resultado corrobora a pesquisa de validação da HLAT-8 para o Brasil, que embora tenha sido realizada com amostra de jovens universitários, também demonstrou a menor pontuação na referida questão ${ }^{(12)}$, assim como na validação da HLAT-8 para a China ${ }^{(16)}$.

A utilização de equipamentos tecnológicos, como acesso à Internet, tem se mostrado uma ferramenta auxiliar no apoio para o conhecimento e autogestão da DM, desde que haja consciência, entendimento e habilidades tecnológicas por parte dos usuários para acessar informações de qualidade $^{(17)}$. Pesquisa transversal, realizada na Etiópia com 423 participantes com doenças crônicas, demonstrou que pessoas mais jovens obtinham maior conhecimento e alta habilidade de LS, principalmente aquelas que utilizavam a Internet diariamente e dispunham de recursos on-line ${ }^{(18)}$.

Um fator que pode comprometer a busca por informações de saúde na Internet é o baixo nível de escolaridade. Essa variável pode estar relacionada com questões cognitivas e dificuldades interpretativas que influenciam no conhecimento sobre a doença, na busca por informações de saúde e na adesão à terapêutica ${ }^{(19)}$. Neste estudo, pôde-se comparar que os percentuais de LS satisfatório e conhecimento adequado foram maiores nos participantes com mais de quatro anos de escolaridade (LS: 17; 65,4\% versus 9; 34,6\% e conhecimento da doença: 16; 61,5\% versus 10 ; 38,5\%) (Tabela 3). Achados semelhantes foram encontrados na validação do SKILLD para a língua portuguesa do Brasil, que apresentou correlação positiva da escolaridade com o conhecimento da DM $(6,6 \pm 5,1, \mathrm{p}=0,003)^{(13)}$.

Verificou-se que o conhecimento adequado e o LS satisfatório foram maiores nos participantes com mais de cinco anos de diagnóstico (16; 64,0\% versus 9; 36,0\%, para ambas as variáveis) (Tabela 3). Resultado que vai ao encontro da validação transcultural do instrumento SKILLD para o Brasil, com 129 idosos com DM, que obteve correlação positiva do conhecimento da doença com o tempo de diagnóstico $(16,5 \pm 8,8, \mathrm{p}=0,022)^{(13)}$. Pessoas com maior tempo da doença estão mais expostas às informações de saúde e tratamento, que possibilitam o desenvolvimento de habilidades e conhecimentos ao longo do tempo ${ }^{(19)}$. 
Todavia, pesquisa transversal realizada em Montes Claros, Minas Gerais, com 353 participantes com DM2, mostrou que 54,9\% tinham tempo de diagnóstico maior de cinco anos e $65,1 \%$ eram sedentários ${ }^{(20)}$. Neste estudo, 91,0\% $(n=30)$ não praticavam atividade física regularmente, fator que pode contribuir para o sobrepeso e o alto risco para o desenvolvimento do pé diabético. Entretanto, observou-se que 50,0\% $(\mathrm{n}=8)$ dos participantes com IMC $\geq 30 \mathrm{~kg} / \mathrm{m}^{2}$ apresentavam baixo/moderado risco para o desenvolvimento de complicações nos pés, sendo necessário investigar outros fatores associados a este achado.

Contrapondo esses achados, pesquisa transversal com 69 participantes com DM mostrou que IMC $>30 \mathrm{~kg} / \mathrm{m}^{2}$ aumenta em 13\% o risco para o desenvolvimento do pé diabético ${ }^{(21)}$. Outro estudo transversal de base institucional, realizado com 279 adultos com DM, na Etiópia, identificou que 13,6\% tinham ulcerações nos pés, e, por meio de análise logística multivariada, essa lesão foi correlacionada com DM2, sobrepeso e obesidade. O IMC elevado é um fator que aumenta a pressão de apoio nos pés e diminui a circulação sanguínea nas extremidades inferiores, elevando o risco para o desenvolvimento de complicações ${ }^{(4)}$. Outro fator que pode responder à associação dessas variáveis com o desenvolvimento do pé diabético é a dificuldade do controle glicêmico em longo prazo ${ }^{(22)}$.

Revisão sistemática, do tipo metanálise, com o objetivo de determinar a associação entre LS, pé diabético, fatores de risco e o autocuidado com os pés, apresentou resultados controversos frente à insuficiência de dados nos estudos, majoritariamente transversais de baixa evidência ${ }^{(23)}$. Esses achados alertam que, focar somente na LS, pode não ser eficaz na redução dos fatores de risco para o pé diabético, sendo necessários estudos longitudinais robustos para evidenciar essa lacuna ${ }^{(14,23)}$.

Ao considerar a variável renda per capita, verificou-se que os participantes com valores $\leq 1.045,00$ reais obtiveram conhecimento inadequado $(n=13 ; 56,5 \%$ versus $n=10 ; 43,5 \%)$ e maior risco para o pé diabético $(n=15 ; 65,2 \%$ versus $n=8 ; 34,8 \%$ ) quando comparados àqueles portadores de conhecimento adequado e LS satisfatório. Este fato pode ser explicado pela dificuldade de acesso aos serviços e às informações de saúde direcionados para a prevenção das complicações da $\mathrm{DM}^{(24)}$. Para tanto, deve-se adequar as organizações e os sistemas de saúde para o cuidado integral e multidisciplinar, além da capacitação para profissionais e familiares, com vistas a melhorar a educação em DM e prevenir o pé diabético ${ }^{(1)}$.

A educação em DM para os pacientes e seus familiares deve ser instigada desde o diagnóstico, a fim de compartilhar conhecimentos e informações para o desenvolvimento de habilidades para o autocuidado. Observou-se que a maior média da HLAT-8 foi no fator estrutural IS, relativo ao fornecimento de informações ou à ajuda de familiares e amigos para elucidar as dúvidas de saúde. Este resultado expressa o potencial da rede de apoio social, que pode ser fortalecida pela aproximação dos diversos profissionais, setores, familiares e amigos na intenção de monitorar e controlar a doença e, consequentemente, postergar as complicações da DM.

Para este estudo, aplicou-se testes de confiabilidade de alfa de Cronbach para os instrumentos de conhecimento e LS, em razão de terem sido validados em populações diferentes da amostra do estudo. O valor obtido para o SKILLD $(0,756)$ revelou boa aplicabilidade para adultos. Entretanto, a confiabilidade da HLAT-8 $(0,511)$ mostrou-se mediana, uma vez que a literatura aponta como ideal, índices acima de $0,7^{(25)}$. Salienta-se que, na validação da HLAT-8 para o Brasil, o alfa de Cronbach foi de 0,74; entretanto, a escala foi aplicada a 472 jovens adultos, amostra superior à pesquisada neste estudo.

Considera-se como limitações deste estudo o baixo número de participantes, que comprometeu a análise estatística e a abordagem transversal, o que não permite a generalização dos resultados.

As contribuições deste estudo para a área da Enfermagem consistem no reconhecimento de fatores associados aos níveis de LS, conhecimento da doença e risco para o pé diabético, pela aplicação de instrumentos validados, fornecendo um arcabouço para a formulação de estratégias 
direcionadas ao controle e à prevenção das complicações da doença, principalmente para as pessoas com baixa renda e escolaridade. Reforça-se a necessidade de estudos futuros que investiguem a dificuldade de entendimento das informações e acesso aos serviços preventivos de saúde, a fim de otimizar as políticas públicas para as pessoas com DM.

\section{Conclusão}

Os resultados demonstraram que níveis satisfatórios de LS estão associados com o conhecimento adequado sobre a doença. Entretanto, ao relacionar essas variáveis com o risco para pé diabético, não houve significância estatística. Os dados também mostraram que as pessoas com menor escolaridade, baixa renda per capita e menor tempo de diagnóstico requerem maior atenção dos serviços primários, com vistas a promover conhecimento e LS, fundamentais na estruturação de ações educativas voltadas para as mudanças no estilo de vida e na prevenção das complicações relacionadas à DM.

Mensurar os níveis de LS, conhecimento da doença e risco para pé diabético permitiu identificar fatores que favorecem ou interferem na autonomia da pessoa com DM para o autocuidado. Os achados, adquiridos por meio dos instrumentos, possibilitam o desenvolvimento de estratégias educativas voltadas para as lacunas do conhecimento sobre a doença e o acesso a informações de saúde e para o fortalecimento da rede de apoio social, com vistas a instrumentalizar a pessoa para a tomada de decisão diária.

A confiabilidade dos instrumentos Eight-Item Health Literacy Assessment Tool e Spoken Knowledge in Low Literacy Patients with Diabetes foi favorável para aplicação em população adulta. Deve-se incentivar o uso desses instrumentos de avaliação, para identificar os fatores que comprometem o autocuidado da pessoa com DM.

Sugere-se estimular pesquisas sobre a temática, com métodos mais robustos e com maior número de participantes, a fim de identificar os fatores associados ao desenvolvimento do pé diabético. Parte dos estudos que tratam dessa temática são do tipo transversal e de baixa evidência, comprometendo os dados e mantendo a lacuna sobre o tema.

\section{Colaborações:}

1 - concepção, projeto, análise e interpretação dos dados: Robson Giovani Paes, Maria de Fátima Mantovani e Ângela Taís Mattei da Silva; 2 - redação do artigo e revisão crítica relevante do conteúdo intelectual: Robson Giovani Paes, Maria de Fátima Mantovani, Ângela Taís Mattei da Silva, Christian Boller, Saimon da Silva Nazário e Elaine Drehmer de Almeida Cruz;

3 - aprovação final da versão a ser publicada: Robson Giovani Paes e Maria de Fátima Mantovani.

\section{Referências}

1. Sociedade Brasileira de Diabetes. Diretrizes Sociedade Brasileira de Diabetes 2019-2020 [Internet]. São Paulo (SP); 2019 [cited 2021 Jan 9]. Available from: http://www.saude.ba.gov.br/wpcontent/uploads/2020/02/Diretrizes-SociedadeBrasileira-de-Diabetes-2019-2020.pdf

2. Silva JMTS, Haddad MCFL, Rossaneis MA, Vannuchi MTO, Marcon SS. Fatores associados à ulceração nos pés de pessoas com diabetes mellitus residentes em área rural. Rev Gaúcha Enferm. 2017;38(3):e68767. DOI: 10.1590/19831447.2017.03.68767

3. Pedras S, Carvalho R, Pereira MG. Características sociodemográficas e clínicas de doentes com pé diabético. Rev Assoc Med Bras. 2016;62(2):171-8. DOI: 10.1590/1806-9282.62.02.171

4. Mariam TG, Alemayehu A, Tesfaye E, Meguannt W, Temesgen K, Yetwale F, et al. Prevalence of Diabetic Foot Ulcer and Associated Factors among Adults Diabetic Patients Who Attend the Diabetic Follow-Up Clinic at the University of Gondar Referral Hospital, North West Ethiopia, 2016: Institutional - Based Cross - Sectional Study. J Diabetes Res. 2017:2879249. DOI: 10.1155/ 2017/2879249

5. Papatheodorou K, Banach M, Bekiari E, Rizzo M, Edmonds M. Complications of diabetes 2017. J Diabetes Res. 2018:3086167. DOI: 10.1155/ 2018/3086167 
6. Lael-Monfared E, Tehrani H, Moghaddam ZE, Ferns GA, Tatari M, Jafari A. Health literacy, knowledge and self-care behaviors to take care of diabetic foot in low-income individuals: Application of extended parallel process model. Diabetes Metab Syndr. 2019;13(2):1535-41. DOI: 10.1016/j.dsx.2019.03.008

7. Chehuen Neto JA, Costa LA, Estevanin GM, Bignoto TC, Vieira CIR, Pinto FAR, et al. Letramento funcional em saúde nos portadores de doenças cardiovasculares crônicas. Ciênc saúde colet. 2019;24(3):1121-32. DOI: 10.1590/ 1413-81232018243.02212017

8. Todorovic N, Jovic-Vranes A, Djikanovic B, Pilipovic-Broceta N, Vasiljevic N, Lucic-Samardzija V, et al. Assessment of health literacy in the adult population registered to family medicine physicians in the Republic of Srpska, Bosnia and Herzegovina. Eur J Gen Pract. 2019;25(1):32-8. DOI: $10.1080 / 13814788.2019 .1571579$

9. Malta M, Cardoso LO, Bastos FI, Magnanini MMF, Silva CMFPD. Iniciativa STROBE: subsídios para a comunicação de estudos observacionais. Rev Saúde Pública [Internet]. 2010 [cited 2021 Sep 10];44(3):559-65. Available from: https://www. scielosp.org/pdf/rsp/2010.v44n3/559-565/pt

10. Brasil. Ministério da Saúde. Secretaria de Atenção à Saúde. Departamento de Atenção Básica. Estratégias para o cuidado da pessoa com doença crônica: diabetes mellitus [Internet]. Brasília (DF); 2013. (Cadernos de Atenção Básica, n. 36) [cited 2021 Jan 10]. Available from: https://bvsms.saude. gov.br/bvs/publicacoes/estrategias_cuidado_ pessoa_diabetes_mellitus_cab36.pdf

11. Bimbato LDM, Pereira FLA, Paladini Filho PH, Creimer HP, Silva VM, Tieppo A, et al. Doença arterial obstrutiva assintomática e Índice Tornozelo-Braquial em pacientes com diabetes mellitus tipo 2. Rev Soc Bras Clin Med [Internet]. 2018 [cited 2021 Jan 9];16(1):18-20. Available from: https://www.sbcm.org.br/ojs3/index.php/rsbcm/ article/view/319/290

12. Quemelo PRV, Milani D, Bento VF, Vieira ER, Zaia JE. Literacia em saúde: tradução e validação de instrumento para pesquisa em promoção da saúde no Brasil. Cad Saúde Pública. 2017;33(2): e00179715. DOI: 10.1590/0102-311X00179715

13. Souza JG, Apolinario D, Farfel JM, Jaluul O, Magaldi RM, Busse AL, et al. Aplicabilidade do Spoken Knowledge in Low Literacy Patients with Diabetes em idosos brasileiros. Einstein
(São Paulo). 2016;14(4):513-9. DOI: 10.1590/ S1679-45082016AO3747

14. Chen P, Callisaya M, Wills K, Greenaway T, Winzenberg T. Associations of health literacy with risk factors for diabetic foot disease: a cross-sectional analysis of the Southern Tasmanian Health Literacy and Foot Ulcer Development in Diabetes Mellitus Study. BMJ Open. 2019;9(7): e025349. DOI: 10.1136/bmjopen2018-025349

15. Hu J, Amirehsani KA, McCoy TP, Wallace DC, Coley SL, Zhan F. Reliability and Validity of the Spoken Knowledge in Low Literacy in Diabetes in Measuring Diabetes Knowledge Among Hispanics With Type 2 Diabetes. Diabetes Educ. 2020;46(5): 465-74. DOI: $10.1177 / 0145721720941409$

16. Guo S, Davis E, Yu X, Naccarella L, Armstrong R, Abel $\mathrm{T}$, et al. Measuring functional, interactive and critical health literacy of Chinese secondary school students: reliable, valid and feasible? Glob Health Promot. 2018;25(4):6-14. DOI: 10.1177/ 1757975918764109

17. Jeffrey B, Bagala $M$, Creighton A, Leavey $T$, Nicholls S, Wood C, et al. Mobile phone applications and their use in the self-management of Type 2 Diabetes Mellitus: a qualitative study among app users and non-app users. Diabetol Metab Syndr. 2019;11:84. DOI: 10.1186/s13098019-0480-4

18. Shiferaw KB, Tilahun BC, Endehabtu BF, Gullslett MK, Mengiste SA. E-health literacy and associated factors among chronic patients in a low-income country: a cross-sectional survey. BMC Med Inform Decis Mak. 2020;20(1):181. DOI: 10.1186/s12911-020-01202-1

19. Moraes KL, Brasil VV, Oliveira GF, Cordeiro JABL, Silva AMTC, Boaventura RP, et al. Letramento funcional em saúde e conhecimento de doentes renais em tratamento pré-dialítico. Rev Bras Enferm. 2017;70(1):155-62. DOI: 10.1590/0034-7167-2015-0169

20. Assunção SC, Fonseca AP, Silveira MF, Caldeira AP, Pinho L. Conhecimento e atitude de pacientes com diabetes mellitus da Atenção Primária à Saúde. Esc Anna Nery. 2017;21(4): e20170208. DOI: 10.1590/2177-9465-EAN-20170208

21. Silva OS, Vieira CSA, Gomes LMX, Barbosa TLA. Grau de risco do pé diabético na atenção primária à saúde. Rev Enferm UFSM. 2020;10:e78. DOI: $10.5902 / 2179769242614$ 
22. Thakur JK, Kumar R, Basu D, Hansda K, Munshi BD, Chakraborty SN, et al. Prevalence of Diabetic Foot Syndrome and Its Determinants among Type 2 Diabetes Mellitus patients attending Integrated Diabetes \& Gestational Diabetes Clinic of a Tertiary Health Care Level Hospital of Eastern India. IOSR J Dent Med Sci. 2019;18(1):24-9. DOI: $10.9790 / 0853-1801092429$

23. Chen PY, Elmer S, Callisaya M, Wills K, Greenaway TM, Winzenberg TM. Associations of health literacy with diabetic foot outcomes: a systematic review and meta-analysis. Diabet Med. 2018;35(11);1470-9. DOI: 10.1111/dme.13694
24. Moura NS, Lopes BB, Teixeira JJD, Oriá MOB, Vieira NFC, Guedes MVC. Alfabetização em saúde e autocuidado em pessoas com diabetes mellitus tipo 2. Rev Bras Enferm. 2019;72(3):700-6. DOI: 10.1590/0034-7167-2018-0291

25. Espinoza SC, Novoa-Muñoz F. Ventajas del alfa ordinal respecto al alfa de Cronbach ilustradas con la encuesta AUDIT-OMS. Rev Panam Salud Publica. 2018;42:e65. DOI: 10.26633/RPSP.2018.65

Recebido: 26 de agosto de 2021 Aprovado: 25 de novembro de 2021

Publicado: 27 de janeiro de 2022

A Revista Baiana de Enfermagem utiliza a Licença Creative Commons - Atribuição-NãoComercial 4.0 Internacional. https://creativecommons.org/licenses/by-nc/4.0/

Este artigo é de acesso aberto distribuído sob os termos da Licença Creative Commons (CC BY-NC). Esta licença permite que outros remixem, adaptem e criem a partir do seu trabalho para fins não comerciais. Embora os novos trabalhos tenham de lhe atribuir o devido crédito e não possam ser usados para fins comerciais, os usuários não têm de licenciar esses trabalhos derivados sob os mesmos termos. 